\title{
The Dynamic Developing Strategies of the Construction of Self-Management Learners' Identity in Online Teaching Space
}

\author{
Xiaoliang Xiong", Jiapei Wang, Huxiao Li \\ Wuhan Technology and Business University, Wuhan, Hubei, China \\ *Corresponding Author.
}

\begin{abstract}
With the implementation of "three accesses and two platforms" (three accesses refer to the access to Internet for each school and college, access to quality educational resources for each class, access to online learning space for each student; two platforms refer to the online platform for educational resources and the online platform for educational management), the online teaching space with more abundant subject knowledge material has become a new teaching field of curriculum reform. Its effectiveness depends on the multiple effects of value pursuit, identity, teaching system, teaching culture, teaching personality and learning style, with students' identity as the subjective initiative factor. In view of the fact that the purpose of education is the premise and foundation of students' identity, this paper applies the methods of sociology and pedagogy to explain the influence of the value orientation of students' identity and the socialization process of learners' development so as to illustrate the dynamic developing strategies of the construction of self-management learners' identity in online teaching space on the basis of the building, developing, formatting and maintenance of emotional education of teaching.
\end{abstract}

Keywords:Online Teaching Space, Value Orientation, Learner's Identity, Technology and Education

\section{Introduction}

In 2019, as the CPC (Communist Party of China) Central Committee and the State Council of China issued "China's Education Modernization 2035" and in 2020, the Ministry of Education issued "The Guideline of the Online Teaching Organization and Management for Higher education during the Epidemic Prevention and Control", "Education Modernization", as the great question of our time, has aroused more and more attention. Universities, colleges and teachers are thinking about the question "what kind of people should be cultivated under modern education" Each parent is also wondering "what kind of people can be cultivated under modern education?" UNESCO's International Commission for Futures of Education points out that "the status and role of learners is an important reference standard for the determination of the nature, value and ultimate purpose for any educational system."[1]For colleges and universities, in order to take the initiative to grasp the opportunity of network communication and communication media popularization, so as to deal with the challenges brought by the personalized and pluralistic lifelong learning society, the key measures are to promote the transformation of "students" to "learners" and to construct students' identity as learners. Learners' Model, Students' Identity and the Definition of Learning Ability and other relevant codes and standards concerning online education, developed by the Technical Sub-Committee on Educational Technology of the National Committee for Standardization of Information Technology, are also listed in the category of learners. There is no doubt that the orientation of the educated, that is, the identity and cultivation orientation of the students, is the present situation and future pursuit of the smooth operation of the whole education system. Before putting forward the construction of the learner's identity, we still need to explore the main value orientation of the teaching content.

At present, most of the literature research related to online education mainly focuses on the selection, organization and interpretation, navigation design, development and implementation of the teaching content of online courses. This typical technical perspective neglects the research on the ontology of online courses, including the identity of learners. With the implementation of the "three accesses and two platforms", the concept of teaching content at all levels and all kinds of schools in China is also being carried out. The online teaching space has become a new teaching field of curriculum reform and the researches on the codes and standards concerning online education also

ISSN: 0010-8189

(C) CONVERTER 2021

www.converter-magazine.info 
has increased day by day. It reflects many problems worth discussing in the smooth operation of online education system, among which students' identity is one of them. In the practice of the world orientation of online curriculum, if the online education system was only regarded as the educational tool of teaching material knowledge orientation, the rich connotation of "autonomy, cooperation and inquiry" advocated by information technology curriculum reform will be concealed, and the orientation of learners' experience and cultural value will be ignored.[2]At present, most of the research on students' identity belongs to the thinking mode of "science world" and "book world". The development, of information technology under the online teaching space, of students' identity authentication system, students' identity identification technology, students' identity information collection and all kinds of media reports concerning students' identity in the field of news and legal system are widely excavated, while the researches on the value orientation of online teaching space and learners' identity has been carried out less and less. Therefore, this paper advocates that the teaching content is open and generated, and is chosen, organized and expounded by both teachers and students. The thinking mode of "fusion theory plus value rationality" should be used to rearrange the ontology and practice of learners' identity in the rich and pluralistic cyber- world.

\section{II.Web-based Organization System in the Perspective of Teaching Objective}

With the advent of the information age, the wave of "knowledge proliferation" of online teaching platform is rising year by year, and the random accessibility of online teaching organization system based on knowledge network is becoming more and more prominent. With the development of teaching ideas and curriculum technology implementation, the history of world teaching can be regarded as the history of teaching organization from knowledge to the key components of network organization. The evolution from individual teaching to group teaching even to class teaching helps us to directly understand the diverse creative space of different orientation on the basis of the preset teaching content. Although the relationship between the preset teaching content and the generated teaching content has different demonstration in real education, it cannot be separated from the support of learning environment and practice community. A core topic of educational modernity is still discipline, and the teaching structure tends to show the normative ethics of "you should or you must". This kind of norms and authority from society can effectively achieve specific and operable teaching goals, but it is also easy to cause the plane, linearity, monotony and deficient of educational relations [3].

In 2018, the Ministry of Education issued the "Education Informatization 2.0 Action Plan", which marked the transition of educational informatization into 2.0 era, and the transformation of teaching material discourse system to teacher discourse system and the transformation of teacher discourse system to student discourse system which tends to transform knowledge into intelligence. In the present educational theory and practice, the school encourages teachers to teach students and bring forth new ideas in the way of "knowledge knot". Meanwhile, the teachers encourage the students to apply technological criticism to promote differentiated teaching which demonstrates a consultation orientation of "I can or I could". These requirements have a contradiction with the unified standard of the teaching mode and evaluation of established linear organization under the request of Normative Ethics, which has objectively led to the confusion of the teachers, as shown in Table 1. In order to get rid of the above confusion, many educational theory and practice researchers redefined the learning environment around the educational application of new technology, and introduce the network organization into teaching to obtain the orderly collection of knowledge hyperplasia on the premise of multi-space fusion.

ISSN: 0010-8189

(C) CONVERTER 2021

www.converter-magazine.info 
Table 1 Knowledge Hyperplasia from Different Teaching Objectives

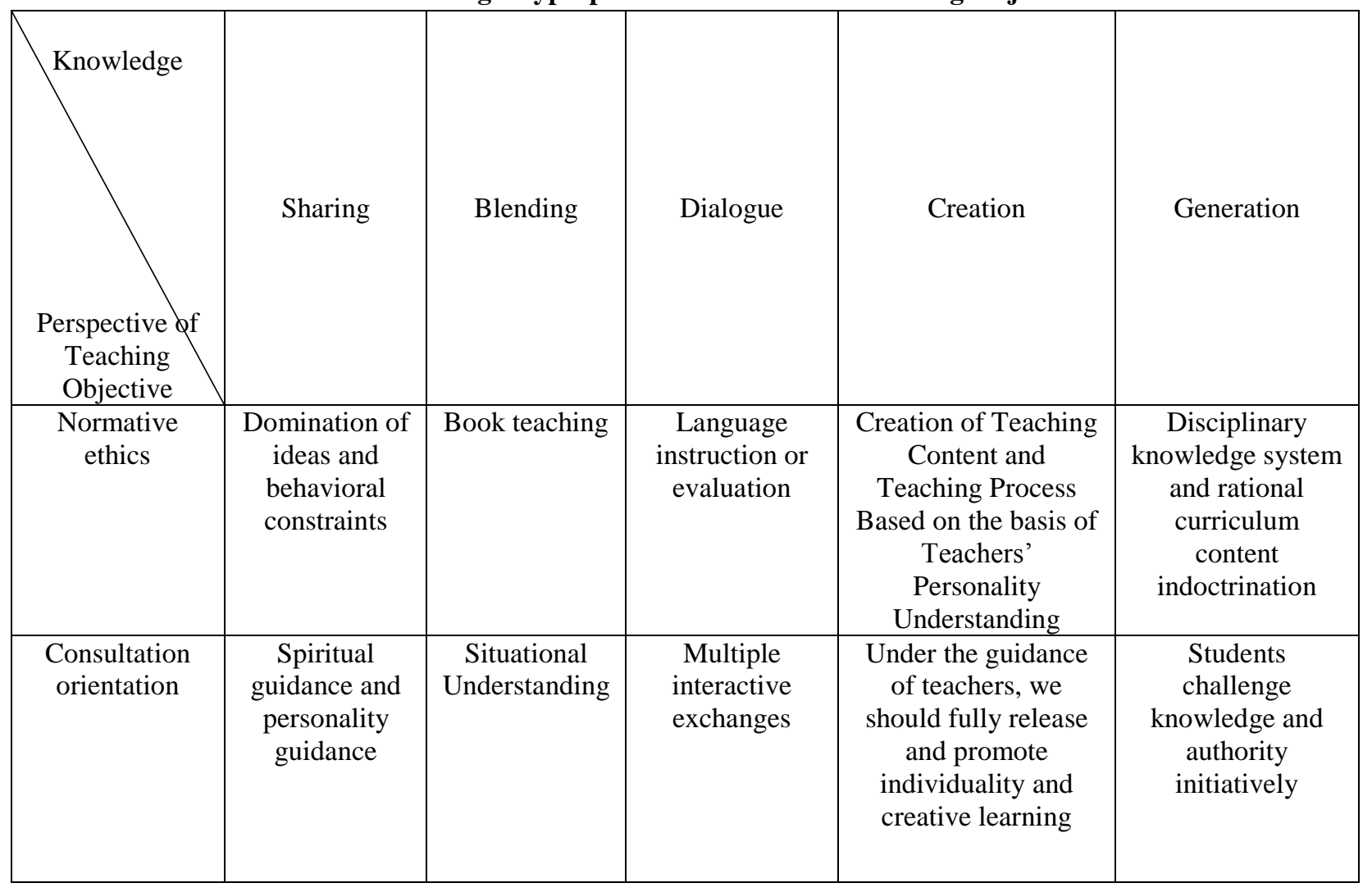

The historical facts of teaching development indicate that it is a direct need to promote the informatization of education and construct the learning system to realize the learning goal through the creation of learning resources and environment and to guide students to inquire knowledge and truth unconditionally and freely.[4]However, we regret to see that the learning process has not yet become a process for learners to obtain real personality independence and individual spiritual freedom under the guidance of the teaching goal of knowledge. The exploration and discovery of learner's identity should focus on teaching and learner's life from the two dimensions of "human's classics and weft" as a whole. Modern teaching, whether in China or in foreign countries, the latitude and longitude of human nature has not been fully taken into account. Consequently, the result is naturally unable to achieve the true comprehensive and harmonious "teacher-student chorus" in cognition, emotion, thinking, personality and other aspects [3]. Therefore, we should carefully examine the final purpose of the deep integration of information technology and the "situational, collaborative and interactive practice" of teaching.

The Postmodern Theory holds that network organization provides the possibility for the full development of students' personality and the integration of large-scale collective teaching because of its dynamic interaction characteristics. These research results can be used in the research of meaningful learning and efficient learning in online teaching space and can provide experience for the online courses to exert the learners' subjectivity. In the process of teaching, the structure network and activity network of subject and object constitute four logical system networks, namely, static "knowledge network", "cognitive network" and dynamic "teaching network" and "learning network". The development area of smooth teaching process in the revolution and informatization of education and teaching is the connection between knowledge logic and cognitive logic and the connection between teaching network and learning network. Hence, the knowledge structure element of the horizontal boundary and the logical order of the vertical boundary of the teaching network organization are important ways for the orderly change of learners' identity in the teaching system.

ISSN: 0010-8189 


\section{Teaching Organization System from the Perspective of Knowledge Classification}

As knowledge upgrading in the new media and new technology era accelerates step by step, students are in the vast network of information resources. The most critical problem is how to effectively obtain the preset and relevant high-quality learning resources so as to generate the internalization channels for knowledge migration. The biggest challenge of knowledge screening, dissemination, distribution, accumulation and development is to study the value source of knowledge in order to answer the question what kind of knowledge is most valuable and whose knowledge is most valuable, and then to find the methods and strategies to transform, generate and construct knowledge, and accordingly to realize effective learning.

Spencer points out in Education that it is necessary to have knowledge selection because students do not have enough time and energy to master all the knowledge of human society. As for the question "what knowledge is the most valuable?" Spencer's answer is science. Nowadays, researchers of educational theory and practice also tend to the fact that "scientific knowledge is the most valuable knowledge". As a result, it is generally considered that classifying and organizing knowledge from the following three levels, different layers of science, different layers of life and different layers of values, is the basic hypothesis of effective design of teaching organization system.[5]In addition to the credibility of these knowledge, the material package in the communication activities between teachers and students in the classroom playing a role in guiding the teaching topic should also take into account the validity of knowledge, that is, the structure of knowledge nodes. By structuring the types of knowledge involved in teaching and their representations to maintain the effectiveness and order of knowledge internalization and proliferation among the multi-level subjects in the real teaching situation are the foundation for improving the boundary of teaching network organization.

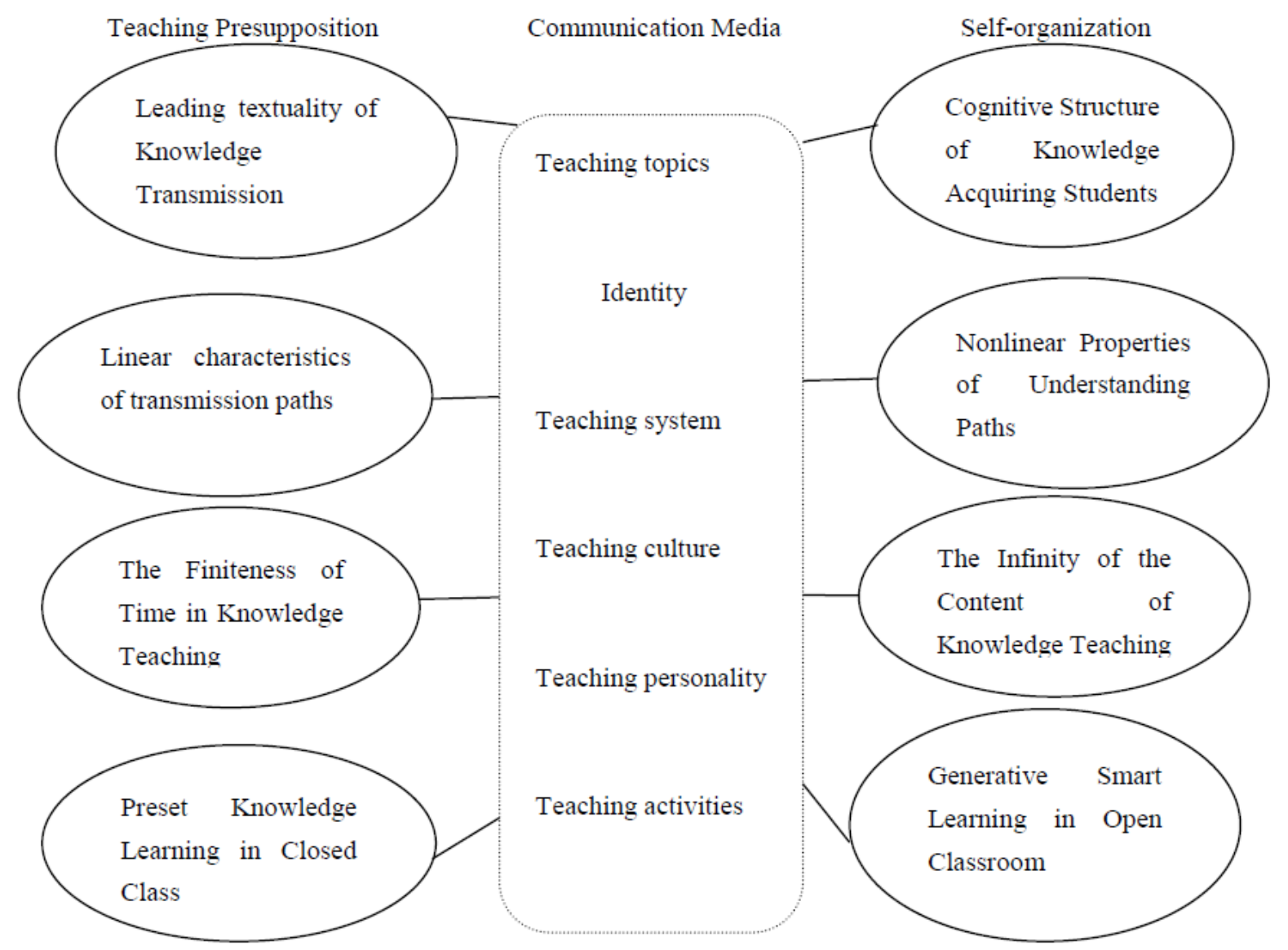

Fig 1: Relationship between Teaching Presupposition and Learning Self-organization.

ISSN: 0010-8189

(C) CONVERTER 2021 
The question that whose knowledge is the most valuable needs to draw lessons from the theory of teaching elements. The integration between "situational knowledge of online teaching and background knowledge of students' learning and"textual knowledge of curriculum" should not only reflect the problem of where to organize, but also reveal the problem of how to organize. In this kind of online curriculum development, the presupposition organization of teachers and the self-organization of students form the "normative" world of teaching explicitly and implicitly,as shown in Figure 1. Identity is the main subjective initiative fact to support the world, while the teaching system is the external statute, and the teaching culture is the value guidance. When any party transcends its own boundaries, it will have an impact on the internalization of students' learning knowledge, that is, the question that how the teaching is organized. Accordingly, the research of presupposition organization of teaching and self-organization of learning in online environment should be carried out in a certain framework. The generation of knowledge should be studied from the mutual connection between daily teaching activities and other elements such as identity, teaching system, teaching culture, teaching personality and so on. Of course, as far as the specific knowledge generation field based on network technology is concerned, it is undoubtedly a mistake to try to seek the one-to-one correspondence between presupposition organization and self-organization. On the contrary, we should use relational thinking to outline the structures of "knowledge logic" and "cognitive logic" in the teaching field, as well as the channels for the effective integration of teaching logic system and learning logic system, so as to perfect and revise the students' learning process and arouse humanistic concern for students' self-organization.

\section{Reflections on the Construction of Learners' Identity in Online Teaching Space}

Starting from the relational thinking of presupposition organization and self-organization, the systematic study of teaching organization in network classroom environment not only can embody the teaching thought of "student subject", but also can reflect the design idea of "cognitive internalization learning". Postmodern Theory holds that active participation is the best learning method, and teaching should cultivate students' awareness of active participation and provide conditions for active participation in order to promote learning effectiveness of students. Yhe relational thinking between learner's identity and network-based learning is a kind of teaching design with constructivism, which aims to measure the way that the subject and object of teaching construct their own cognitive structure, straighten out the order, communication, accretive and understanding of the transformation from knowledge system to cognitive system in teaching system, and internalize knowledge logic system into cognitive logic system with the help of teaching logic channel.

\subsection{The representation and creation of problems is the key to the construction of learners' identity}

Teaching objective, teaching preparation, teaching content, teaching structure and teaching method and strategy design directly point to the expression and creation of teaching problems, due to the internal order, systematisms and coherence of teaching problems. Therefore, we reckon that the order of knowledge transmission and the expression and creation of problems have the same order structure and that the representation of problems have an effect on the orientation and speed of knowledge transmission. Appropriate external problem representation, such as text, number, chart, model, experiment and so on, can not only make the teaching subject aware of the structural types of the problem to reduce the burden of memory of teachers and students, but also activate the cognitive operation of perception to improve the quality of communication between teachers and students. Online teaching space provides convenient conditions for the expression and creation of problems. Multimedia visualization methods such as pictures, video, animation and so on can become the guidance or extension of text resources in some teaching situations that need independent expression of ideas [6]. In the self-organized learning space, learners master their own learning situation and communicate and cooperate with others. If teachers cannot capture these fleeting valuable opportunities to stimulate learning interest, attract students' learning attention and develop their thinking ability through the situation creation and question design of teaching problems, the effect of knowledge transmission will be affected. Therefore, it can be said that whether teachers have the consciousness and the capacity of teaching resources in online teaching space is the guarantee to

ISSN: 0010-8189

(C) CONVERTER 2021

www.converter-magazine.info 
support the construction of learners' identity.

\subsection{Sorting and expressing key words is the core of learners' identity construction}

The functional modules such as personal center, home page, general setting of space, space management, favorites and message board in online teaching space are effective platforms for the combination and expansion of teaching text, in which the sorting of keywords is the entity element of mutual inquiry, reflection, instant and non-instant communication between teachers and students. Keyword expression should pay special attention to the following three problems: right time, order and way of keywords presentation. First, right time of keyword presentation refers to the proper time to find connections between learning tasks, learning resources, learning monitoring and learning evaluation according to the design of knowledge objectives, and to identify the key words that are the most suitable for achieving the goal. Second, order of keyword presentation refers to the order of the process in which the sorting of keywords in the dialogue must be on the stand of students in application of the resources provided by online teaching platform and the advantages of online environment, meanwhile, the sorting of keywords effectively combined the learning of knowledge and the completion of the project implying the answers should take the learners' mindset into account. Third, way of keyword presentation refers to whether the direct oral dialogue, or indirect written speech dialogue between teachers and students should focus on keywords and contradictory questions, step-by-step questions, divergent questions, variant questions with a variety of forms such as rhetorical questions, questioning, continuous questions to stimulate the flexibility of learners' mindset [7].Finally, effective keyword presentation, along with the habit of learners' mindset reflect the idea of "teaching for knowledge" and "learning for knowledge", so as to truly achieve concise and effective communication between teachers and students.

\section{3"Intelligence" is the subject of learners' identity construction}

The current teaching is regarded as a generative activity based on communication and interaction. Whether teachers can effectively guide students to learn depends on whether teachers can consciously help students to train and develop their thinking in the infinite changing of logic sequence reorganization and arrangement activities of knowledge, and then to help students to feel the charm of teaching activities. First, in terms of resource acquisition, students are trained to process the collected information, and instructed to constantly change the content and structure of learning resources to form their own derivative ideas via the production of project works so as to take into account the contradiction between the limitation of teaching time and the infinity of teaching content. Second, in the aspect of resource integration, teachers' teaching activities should not only ensure the achievement of knowledge objectives, but also ensure the understanding of different styles, and encourage students to reconstruct knowledge system by enhancing basic knowledge and expanding learning fields along with the change of learning environment, learning objectives and tasks, so as to alleviate the contradiction between the linear characteristics of transmission and the nonlinear characteristics of understanding.[8]Third, in terms of evaluation resources, there is a contradiction between the leadingtextuality of knowledge and the cognitive structure of students. Since the self-evaluation of students' knowledge value is conducive to the establishment of the status of learning subjects, it is necessary to guide students to multidimensionally think about learning tasks or learning topics and judge the value of learning resources. Fourth, as for selection of resources, it is important to guide students to select proper resources to meet their individual learning needs from the large resources with various forms and fields, so that the organizational strategy of current curriculum can transform from in-class learning to space-time open cross-class learning, accordingly reflecting the concept of adaptive teaching.

\subsection{Subjectivity teaching model is the thrust of learners' identity construction}

The learning process of transforming knowledge into intelligence, as a hierarchical knowledge learning pays more attention to the analysis, integration and evaluation of learning, is a kind of teaching value pursuit that more focus on the meaning of knowledge, that is, the subjective teaching mode with more emphasis on spiritual transformation. Therefore, the positive attitude experience of students in knowledge teaching, such as ability, 
effort, environment, monitoring and self-efficacy, is the thrust of transforming knowledge into intelligence. The experience of educational relationship hidden in the online learning environment and the online curriculum needs to be recognized with a lot of importance. The intrinsic motivation factors of students' learning, such as the awareness of learning values, the initiative of reporting personal or group works, the arrangement of the role and task of students' learning group, should be paid special attention in order to cultivate students' sense of effort to learning. Teachers need to guide students to establish an independent learning view, praise and encourage them promptly according to the active participation shown by the dynamic data of "student participation" in the project learning activities, and train students to grasp the methods of self-learning to improve the learning ability of students as learning subject. It is necessary for teachers to guide students to apply concept map, mind map and other tools to outline their own inquiry learning framework, to guide students to make use of the required content structure or problem-solving framework to generate individual views and living space of thinking, and to enhance students' sense of environment in creating learning situations [9]. Teachers need to provide students with reflective situational knowledge of teachers' teaching, and the language, mindset and thinking strategies and incentives of the editor-in-chief of teaching materials etc., so that develop students' sense of self-monitoring to actively control and regulate their learning progress, time, activities and processes. As a result, teachers can train students' complex mindset in the process of "situation-question-communication-solution", provide the gauge of adjusting and optimizing learning methods and strategies in earning process to enhance their self-efficacy.

\section{Conclusion}

In view of the fact that the purpose of education is the premise and foundation of students' identity, this paperillustrates the dynamic developing strategies of the construction of self-management learners' identity in online teaching space on the basis of the building, developing, formatting and maintenance of emotional education of teaching,such as the representation and creation of problems,sorting and expressing key words, “intelligence", subjectivity teaching model.

\section{Acknowledgments}

This research was financially supported by Hebei Educational Science Planning and Research "Assessment of Job Competence of International Economics and Trade Majors Oriented at the Industrial Development and Construction of Hubei Free Trade Zone" (2019GB129).

\section{References}

[1] David. Carr, translated by $\mathrm{Xu}$ Wu. The Significance of Education. Beijing: China Renmin University Press, 2015

[2] Alain. De. Burton, translated by Chen Guangxing, Nan Zhiguo. Anxiety of identity. Shanghai: Shanghai Translation Publishing House, 2014

[3] Y. L. Jin, Z. Y. Yu,Curriculum Theory (2nd Edition). Beijing: people's Publishing House, 2015

[4] J. Y.Ye, A Historical Sociological Study on the Construction of Teacher Identity. Beijing: Beijing Normal University Press, 2017

[5] Y. F. Liu,the Connotation, Composition and Development of Teachers' Personal Knowledge. Educational Research, 2017(06):101-106

[6] Yuan. Derun, promoting the development of teachers' professional ability with classroom as the carrier: the perspective of the transformation of personal knowledge and practice. Global Education Outlook, 2020(06):81-89

[7] H. X. Li, The Dilemma of Teacher's Curriculum Power and its Management. Educational Science Research, 2020(02):93-96

[8] V. Frunzaru, GARBASEVSCHID. Students' Online Identity Management. Journal of Media Research,2016, 9(1):3-13

[9] Gieser. J. D, A Sociocultural Investigation of Identity: How Students Navigate the Study Abroad Experience. Journal of College Student Development, 2015, 56(6):637-643

ISSN: 0010-8189

(C) CONVERTER 2021 